\title{
Recurrent inhibition of mitochondrial complex III induces chronic pulmonary vasoconstriction and glycolytic switch in the rat lung
}

Olga Rafikova ${ }^{1,2}$, Anup Srivastava ${ }^{1,2}$, Ankit A. Desai $^{2}$, Ruslan Rafikov ${ }^{1,2^{*}}$ (i) and Stevan P. Tofovic ${ }^{3}$

\begin{abstract}
Background: Pulmonary arterial hypertension (PAH) is a fatal disease; however, the mechanisms directly involved in triggering and the progression of PAH are not clear. Based on previous studies that demonstrated a possible role of mitochondrial dysfunction in the pathogenesis of $\mathrm{PAH}$, we investigated the effects of chronic inhibition of mitochondrial function in vivo in healthy rodents.

Methods: Right ventricle systolic pressure (RVSP) was measured in female rats at baseline and up to 24 days after inhibition of mitochondrial respiratory Complex III, induced by Antimycin A (AA, 0.35 mg/kg, given three times starting at baseline and then days 3 and 6 as a bolus injection into the right atrial chamber).

Results: Rodents exposed to AA demonstrated sustained increases in RVSP from days 6 through 24. AA-exposed rodents also possessed a progressive increase in RV end-diastolic pressure but not RV hypertrophy, which may be attributed to either early stages of PAH development or to reduced RV contractility due to inhibition of myocardial respiration. Protein nitration levels in plasma were positively correlated with PAH development in AA-treated rats. This finding was strongly supported by results obtained from PAH humans where plasma protein nitration levels were correlated with markers of PAH severity in female but not male PAH patients. Based on previously reported associations between increased nitric oxide production levels with female gender, we speculate that in females with PAH mitochondrial dysfunction may represent a more deleterious form, in part, due to an increased nitrosative stress development. Indeed, the histological analysis of AA treated rats revealed a strong perivascular edema, a marker of pulmonary endothelial damage. Finally, AA treatment was accompanied by a severe metabolic shift toward glycolysis, a hallmark of PAH pathology.

Conclusions: Chronic mitochondrial dysfunction induces the combination of vascular damage and metabolic reprogramming that may be responsible for PAH development. This mechanism may be especially important in females, perhaps due to an increased NO production and nitrosative stress development.
\end{abstract}

Keywords: Pulmonary hypertension, Glycolytic switch, Mitochondrial complexes, Protein nitration, Metabolomics

\footnotetext{
* Correspondence: ruslanrafikov@email.arizona.edu

${ }^{1}$ Division of Endocrinology, Department of Medicine, The University of Arizona, Tucson, AZ 85724, USA

${ }^{2}$ Department of Medicine, The University of Arizona, Tucson, AZ, USA

Full list of author information is available at the end of the article
}

(c) The Author(s). 2018 Open Access This article is distributed under the terms of the Creative Commons Attribution 4.0 International License (http://creativecommons.org/licenses/by/4.0/), which permits unrestricted use, distribution, and reproduction in any medium, provided you give appropriate credit to the original author(s) and the source, provide a link to the Creative Commons license, and indicate if changes were made. The Creative Commons Public Domain Dedication waiver (http://creativecommons.org/publicdomain/zero/1.0/) applies to the data made available in this article, unless otherwise stated. 


\section{Background}

Pulmonary arterial hypertension is tightly associated with mitochondrial dysfunction $[1,2]$. There are several different mechanisms of mitochondrial dysfunction that have been proposed to be involved in PAH pathogenesis, such as electron transport chain (ETC) dysfunction [3, 4], insufficient mitochondrial biogenesis [5, 6], altered mitochondrial membrane potential [7], increased mitochondrial reactive oxygen species (mROS) production [8, 9], mitochondrial DNA damage [10], and insufficient mitochondrial repair via fission/fusion or mitophagy [11-13]. Most of those mechanisms are also interconnected in many ways. For example, an increased mitochondrial ROS and DNA damage could initiate the processes of fission/fusion, apoptosis or impaired biogenesis. The current work focuses specifically on ETC dysfunction based on its important roles in both ATP production by mitochondrial respiration and as a primary contributor to an increase in mitochondrial ROS. The resulted oxidative stress is known to be strongly incorporated into $\mathrm{PAH}$ pathogenesis due to its ability to induce DNA and protein damage. Finally, ETC dysfunction mediates an increase in the mitochondrial potential that has been previously described to mediate a glycolytic shift [14].

There are several clinical cases reporting that mitochondrial respiratory chain disease is accompanied by a manifestation of primary pulmonary hypertension $(\mathrm{PPH})$ and severe pulmonary hypertension $[15,16]$. The assessment of the respiratory complexes activity indicated a decreased Complex I and III activity in the tissues of these patients (liver tissue upon liver transplantation, skeletal muscle and skin fibroblasts obtained through biopsy). Thus, the inhibition of Complex III activity is a physiologically relevant stimulus to investigate whether ETC inhibition can induce pulmonary hypertension in a pre-clinical model. Mitochondrial respiratory complexes utilize iron-sulfur clusters to transfer electrons from donors to acceptors. It was also reported that the mutations in the iron-sulfur chaperon protein NFU1 have a strong association with pulmonary hypertension development [17]. All seven patients with NFU1 mutation developed fatal encephalopathy and pulmonary hypertension. Assessment of mitochondria respiratory complexes showed a decrease in Complexes I, II and III activities in patients with the NFU1 mutation, thus, pointing on respiratory chain failure as an important contributor in the development of pulmonary hypertension.

ETC can be affected by inhibition of any of its four known complexes. However, inhibition of Complex III was found to induce maximal ROS production and therefore maximal damage to mitochondria [18]. Electrons flow from Complex I or II to Complex III and then Complex IV generating a gradient of protons in the inner membrane. This gradient is utilized by ATP synthase to produce ATP or by uncoupling proteins to produce heat.
Inhibition of Complex III can induce superoxide production in both the intermembrane space and the mitochondrial matrix [19]. Superoxide radical is usually scavenged by a mitochondrial form of superoxide dismutase MnSOD and transformed into less active hydrogen peroxide $\left(\mathrm{H}_{2} \mathrm{O}_{2}\right)$ [20]. However, nitric oxide (NO) produced in the vasculature can also scavenge superoxide to form peroxynitrite, a highly potent oxidant, which may inhibit ROS scavengers by induction of protein nitration and nitrosylation, like MnSOD [21], and initiate many pathophysiological pathways [22, 23].

Indeed, lungs of patients with PAH have possessed a ubiquitous nitrotyrosine staining $[24,25]$, which indicated a high level of peroxynitrite formation in $\mathrm{PAH}$ patients. Caveolin-deficient mice that are exhibited chronic pulmonary hypertension and severe right ventricle (RV) hypertrophy [26] had a high level of protein nitration in the lungs due to persistent endothelial nitric oxide synthase (eNOS) activation. Notably, the treatment of Cav1-/- mice with either an eNOS inhibitor or a superoxide scavenger reversed the $\mathrm{PAH}$ phenotype. In our recent work, we have reported that nitration mediates an over-activation of Akt with subsequent translocation of eNOS to the mitochondria. This, in turn, reduces the mitochondrial respiration and promotes endothelial dysfunction [27].

The primary female hormone estrogen is known to increase NO production due to an increase in both eNOS expression and activity $[28,29]$. The vasodilatory, antiinflammatory, and anti-thrombotic properties of $\mathrm{NO}$ provide the background for an advanced level of cardiovascular protection in females compared to males. At the same time, it could be expected that in the presence of mitochondria dysfunction and increased production of superoxide, the estrogen-mediated elevation of NO bioavailability can turn to be a damaging factor through an enhanced formation of peroxynitrite. Since the nitrosative stress has been linked with PAH development, it is possible that an enhanced protein nitration may explain the higher prevalence of $\mathrm{PAH}$ in females compared to males. Therefore, in this study, we utilized female rats to elucidate the consequences of repetitive inhibition of the ETC.

Under normal conditions, cells mainly rely on oxidative phosphorylation due to a highly efficient nature of mitochondria-mediated ATP production. However, the disease conditions associated with mitochondrial damage limits mitochondria's ability to generate ATP and force the cells to switch to glycolysis [30, 31]. This metabolic shift towards a glycolytic metabolism, known as the Warburg effect, has been described in cancer cells, as well as highly proliferative pulmonary vascular cells $[32,33]$. Importantly, in our previous research, we have confirmed that the metabolic transformation occurs even at the early stage of PAH when the physiological manifestation of the disease, such as RV pressure and hypertrophy, 
are still negligible [34]. Thus, the metabolic alterations significantly precede PAH manifestation and may be considered as a contributing factor for PAH development. The current work confirms that inhibition of the mitochondrial ETC is sufficient to initiate the same metabolic changes as seen in lungs of patients with PAH.

\section{Methods}

\section{A rat model of pulmonary hypertension}

Female Sprague Dawley rats (200-250 g) were obtained from Charles River (Wilmington, MA, USA) and used in this study. Animals were housed at $22{ }^{\circ} \mathrm{C}, 12$-h light cycle, $55 \%$ relative humidity, and had free access to standard rodent food and water. All experiments were performed in accordance with the University of Pittsburgh Institutional guidelines for animal welfare and the Animal Care and Use Committee approved experimental protocols. On the day of Antimycin A injection, the animals were randomized to receive either vehicle or AA, while anesthetized (pentobarbital, $45 \mathrm{mg} / \mathrm{kg}$ i.p.). The PE-50 catheter and Millar catheter were inserted into the right jugular vein, and PE-50 catheter was advanced into right atrium for i.v. AA injection, while the Millar catheter was used to monitor right ventricular systolic pressure (RVSP) and right ventricular diastolic pressure (RVDP) as described previously [21, 35]. Briefly, a customized pressure transducer catheter (SPR-513; Millar Instruments, Houston, TX, USA) was connected to a Millar MPCU-200 pressure-volume conductance system to monitor RV pressure for 30-min after AA injection.

For acute AA treatment (Group AA $30 \mathrm{~min}$ ), $30 \mu \mathrm{l}$ of $0.35 \mathrm{mg} / \mathrm{kg}$ AA dissolved in $55 \%$ ethanol $/ 45 \% 0.9 \%$ saline was injected as a slow bolus injection; the animal was monitored for $30 \mathrm{~min}$ and euthanized. This dose was selected based on preliminary experiments (not shown) and was found to be the lowest effective dose tolerated by rats. A bolus injection given directly into the right atrium was used to maximize the exposure of the pulmonary vasculature to AA and minimize the AA effects on the left ventricle. For chronic studies (Groups 6 days, 12 days and 24 days) after the initial injection of $\mathrm{AA}$, the PE-50 catheter placed into the right jugular vein was fixed to the sternocleidomastoid muscle and advanced subcutaneously to the back of the neck through the incision between the scapulae. The ventral neck incision was closed with the wound clips and the dorsal - with 4-0 silk suture to secure the exterior part of the catheter in place and was used for chronic AA injections on days 3 and 6. The RVSP on days 6, 12 and 24 was measured as described above.

\section{Measurement of mitochondrial respiration}

An XF24 Analyzer (Seahorse Biosciences, North Billerica, MA) was used to measure mitochondrial function in intact cells, in real time. Cells (rat pulmonary artery smooth muscle cells and rat pulmonary artery endothelial cells (Cell biologics)) were seeded into Seahorse Bioscience XF24 cell culture plates at the seeding density of 75,000 cells in $100 \mu \mathrm{l}$ media and allowed to adhere and grow for $16 \mathrm{~h}$ in a $37{ }^{\circ} \mathrm{C}$ humidified incubator with $5 \%$ CO2. Cells were exposed to Antimycin A $(10 \mu \mathrm{M})$ through the injection port $\mathrm{A}$ of the instrument, and continuous measurements of oxygen consumption rate (OCR) were made in media. The total protein was measured by BCA method, used for normalizing the data. The resultant OCR profile provides information on mitochondrial respiratory responses to AA.

\section{Chemicals}

Antimycin A and ethanol for preparation of the stock solution $(10 \mathrm{mg} / \mathrm{ml})$ were purchased in Sigma-Aldrich (St. Louis, MO).

\section{Histological analysis}

At the end of experiments, the trachea catheter was connected to a Harvard rodent ventilator (Model 683; Harvard Apparatus, South Natick, MA, USA), the thorax was opened, and the lungs were flushed with saline $(0.9 \%$ sodium chloride) to remove the blood from the pulmonary vessels. Animals were euthanized by the anesthetic overdose (pentobarbital $200 \mathrm{mg} / \mathrm{kg}$ ), and the heart and lungs were dissected and weighed. The right ventricle (RV) free wall was separated from the left ventricle (LV), and the septum (S) and the Fulton index $(\mathrm{RV} / \mathrm{LV}+\mathrm{S})$ was calculated. The total wet lung weight was measured and normalized by the body weight of the animal. Lower right lobe was removed, weighed, and stored for $18 \mathrm{~h}$ at $65 \mathrm{C}^{0}$. The dry weight was measured and wet-to-dry weight ratio calculated. One lung was immersed in 10\% buffered formalin for at least $72 \mathrm{~h}$ before being embedded, whereas the other was stored at $-80{ }^{\circ} \mathrm{C}$ and used for biochemical studies. Four-micrometer serial tissue sections from paraffinembedded lungs were dewaxed and stained with hematoxylin and eosin (H\&E), Verhoeff's-Van Gieson (VVG) and Pentachrome staining for histological and morphometric assessment. Perivascular cuffs area was blindly calculated by subtraction of Media vessel area from the Cuff area. The data from Day 12 and 24 were based on vessels size $50-100$ and 100-300 um. The Aperio ScanScope XT system (Aperio Inc., Vista, CA, USA) was used to scan entire glass slides at $20 \times$ magnification, and Aperio ImageScope and ImageJ software were used to analyze digital slides.

\section{Plasma protein nitration in rat and human samples}

Dot-blot analysis was performed to measure the total level of plasma protein nitration as previously described [21] 
with modifications. Briefly, $1 \mu \mathrm{l}$ of diluted plasma samples were spotted onto a nitrocellulose membrane (Bio-Rad). The membrane was then allowed to dry overnight at room temperature, blocked with $1 \%$ of BSA in TBS containing $0.1 \%$ Tween 20 for $1 \mathrm{~h}$, and incubated with antibodies specific for either 3-nitrotyrosine (3-NT; 1:2000; Calbiochem) for $2 \mathrm{~h}$ at room temperature. After being washed three times, the membranes were incubated with secondary antibody, washed, visualized using the chemiluminescent procedure, recorded using a Li-Cor Odyssey FC Imager and analyzed using IS Image Studio 5.0 software. For measuring plasma protein nitration in rat samples, the obtained signal was normalized per total plasma protein by using the $1 \mu \mathrm{g}$ of plasma protein/ $\mu \mathrm{l}$ of spotted sample in all rat samples. For patient samples, the obtained protein nitration signal was normalized to the total plasma protein amount measured by Ponceau S stain and quantified by Image J software.

\section{Human subject's blood collection}

All subjects provided written consent to participate in this study with the approval of the University of Arizona (UA) institutional human subjects review board. The study cohort consisted of patients with a right heart catheterization (RHC)-confirmed diagnosis of Group I $\mathrm{PAH}$ and were prospectively recruited from the UA PAH clinic from 2013 to 2015. Blood from 12 male patients and 29 female patients was drawn during outpatient clinic visits or during an electively scheduled RHC (from an antecubital vein). Care was taken to standardize blood sample collection, preparation and storage at $-80{ }^{\circ} \mathrm{C}$ as described [36].

\section{Glycolytic intermediates analysis}

Data were acquired using the West Coast Metabolomics Center at UC Davis. Briefly, Restek corporation Rtx-5Sil MS column was used with helium mobile phase at temperature interval $\left(50-330{ }^{\circ} \mathrm{C}\right)$ and flow-rate: $1 \mathrm{~mL} \mathrm{~min}^{-1}$. Injection volume was $0.5 \mu \mathrm{L}$ at $50{ }^{\circ} \mathrm{C}$ ramped to $250{ }^{\circ} \mathrm{C}$ by $12{ }^{\circ} \mathrm{C} \mathrm{s}^{-1}$. Oven temperature started at $50{ }^{\circ} \mathrm{C}$ for $1 \mathrm{~min}$, then ramped at $20{ }^{\circ} \mathrm{C} \mathrm{min}{ }^{-}$ ${ }^{1}$ to $330^{\circ} \mathrm{C}$, and held constant for $5 \mathrm{~min}$. Mass spectrometry analysis was done on a Leco Pegasus IV mass spectrometer with unit mass resolution at 17 spectra $\mathrm{s}^{-1}$ from 80 to $500 \mathrm{Da}$ at $-70 \mathrm{eV}$ ionization energy and $1800 \mathrm{~V}$ detector voltage with a $230{ }^{\circ} \mathrm{C}$ transfer line and a $250{ }^{\circ} \mathrm{C}$ ion source. Raw data files were preprocessed directly after data acquisition and stored as ChromaTOF-specific ".peg files, as generic *.txt result files and additionally as generic ANDI MS *.cdf files. ChromaTOF vs. 2.32 was used for data preprocessing without smoothing, $3 \mathrm{~s}$ peak width, baseline subtraction just above the noise level, and automatic mass spectral deconvolution and peak detection at signal/noise levels of 5:1 throughout the chromatogram. Apex masses were reported for use in the BinBase algorithm. Result ".txt files were exported to a data server with absolute spectra intensities and further processed by a filtering algorithm implemented in the metabolomics BinBase database.

The data were represented as peak heights for the quantification ion ( $\mathrm{mz}$ value) at the specific retention index. Final data were plotted in the GraphPad Prism software.

\section{Statistical analysis}

Results are presented as Mean \pm SEM. Statistical analyses were performed using GraphPad Prism V. 4.01 software. Group comparisons were carried out using one-factor nested (hierarchical) analysis of variance (1F-ANOVA). If ANOVA indicated a significant difference among the means, specific comparisons were made with NewmanKeulus multiple comparison tests. Alternatively, the unpaired $t$-test between two specific animal or cell treatments groups was used. The statistical analysis used in each figure and the number of replications are indicated in the figure legend. A value of $P<0.05$ was considered significant. An analysis of the correlation between patient plasma 3NT levels and cardiac output or Pulmonary Vascular Resistance (PVR) and animal lung glucose levels and RVSP were performed using two-tail test of the linear regression model. The Pearson coefficient and $P$ values calculated by GraphPad Prism V. 4.01 software are presented on the corresponding figures.

\section{Results}

Repetitive inhibition of mitochondrial respiration induces a sustained increase in right ventricle pressure

The injection of AA-induced a mild but significant increase in RVSP even 30 min after the first injection (Fig. 1a left panel). However, this acute vasoconstrictive response was lost on day 3 after the first injection (Fig. 1a left panel). The second dose, given on day 3 , promoted a prolonged increase in RVSP even 3 days after the second injection (or day 6 since initiation of treatment). Finally, the third dose injected on day 6 ensured the RVSP to stay significantly increased and continue to elevate during the next 18 days after the last injection (or on days 12 and 24 since initiation of study) (Fig. 1a right panel). While we suggest that the acute AA response may be due to an ability of superoxide to scavenge $\mathrm{NO}$ or induce the calcium signaling in the smooth muscle cells [37], we proposed that in the more advanced stages, and especially on day 12 and day 24, other mechanisms are responsible for chronic vasoconstriction. Some of these mechanisms were uncovered in this study.

Surprisingly, while chronic AA treatment induced an increase in RV pressure, the Fulton index and RV normalized on body weight in all experimental groups remained unchanged (Fig. 1b). At the same time, although the AA did not induce an RV hypertrophy, the RV diastolic function was found to be affected. Thus, the RV end Diastolic Pressure (RVDP) showed a progressive increase and 

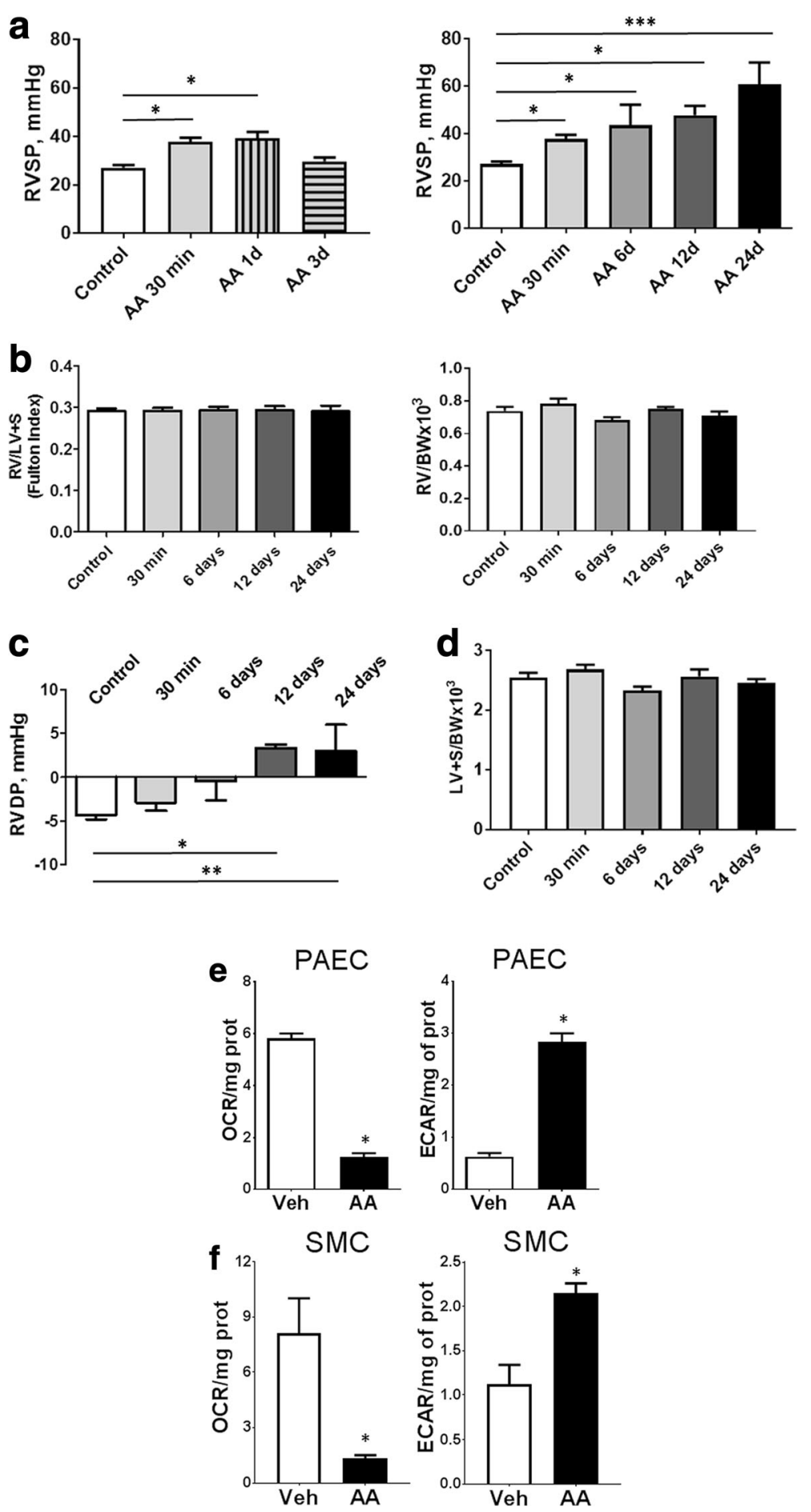

Fig. 1 Acute and chronic right ventricle hemodynamics and remodeling in Antimycin A-treated rats. RV systolic (a) pressure in untreated Controls and acutely AA-treated rats $30 \mathrm{~min}, 1$ and 3 days after AA $(0.35 \mathrm{mg} / \mathrm{kg})$ injection show the acute and reversible effect of one dose of AA on pulmonary pressure (left panel). Right panel represents RV systolic pressure in chronically treated rats. 6, 12 and 24 days after AA injection show a progressive chronic pulmonary vasoconstriction and RV dysfunction. There was no evidence of RV hypertrophy during the first 24 days of AA treatment (b). End diastolic RV pressure indicates significant elevation at day 12 and 24 (c). There is no indication of LV hypertrophy (d). AA (10 $\mu M)$ treatment of PAEC (e) or SMC ( $\mathbf{f}$ ) results in a 6-fold decrease in OCR. Decrease in OCR was accompanied with an increase in glycolysis as indicated by ECAR. Data are mean \pm SEM, $N=4-13$ per group. ${ }^{*} P<0.05$ vs. Control/Nehicle in 1-way ANOVA or t-test for two groups 
became significantly higher by day 12 and day 24 compared to controls (Fig. 1c). Importantly, Left Ventricle (LV) was not affected throughout the course of the study (Fig. 1d).

By treating rat Pulmonary Artery Endothelial Cells (PAEC) and Pulmonary Artery Smooth Muscle Cells (PASMC) with the same dose of AA used to initiate pulmonary vasoconstriction in vivo, we have confirmed that this dose AA is capable to efficiently inhibit mitochondrial respiration in pulmonary vascular cells. Our data from Seahorse XF analyzer indicated a 6-fold decrease in Oxygen Consumption Rate (OCR) in both PAEC and PASMC (Fig. 1e, f). Opposing to OCR, the ExtraCellular Acidification Rate (ECAR) that reflects lactate production due to glycolysis was increased by 6 -fold in PAECs and 2-fold in PASMC. These data indicate that PAEC are more readily switch to glycolysis, while PASMC with inhibited mitochondrial respiration are less compensated in the energy demand compared to endothelial cells.

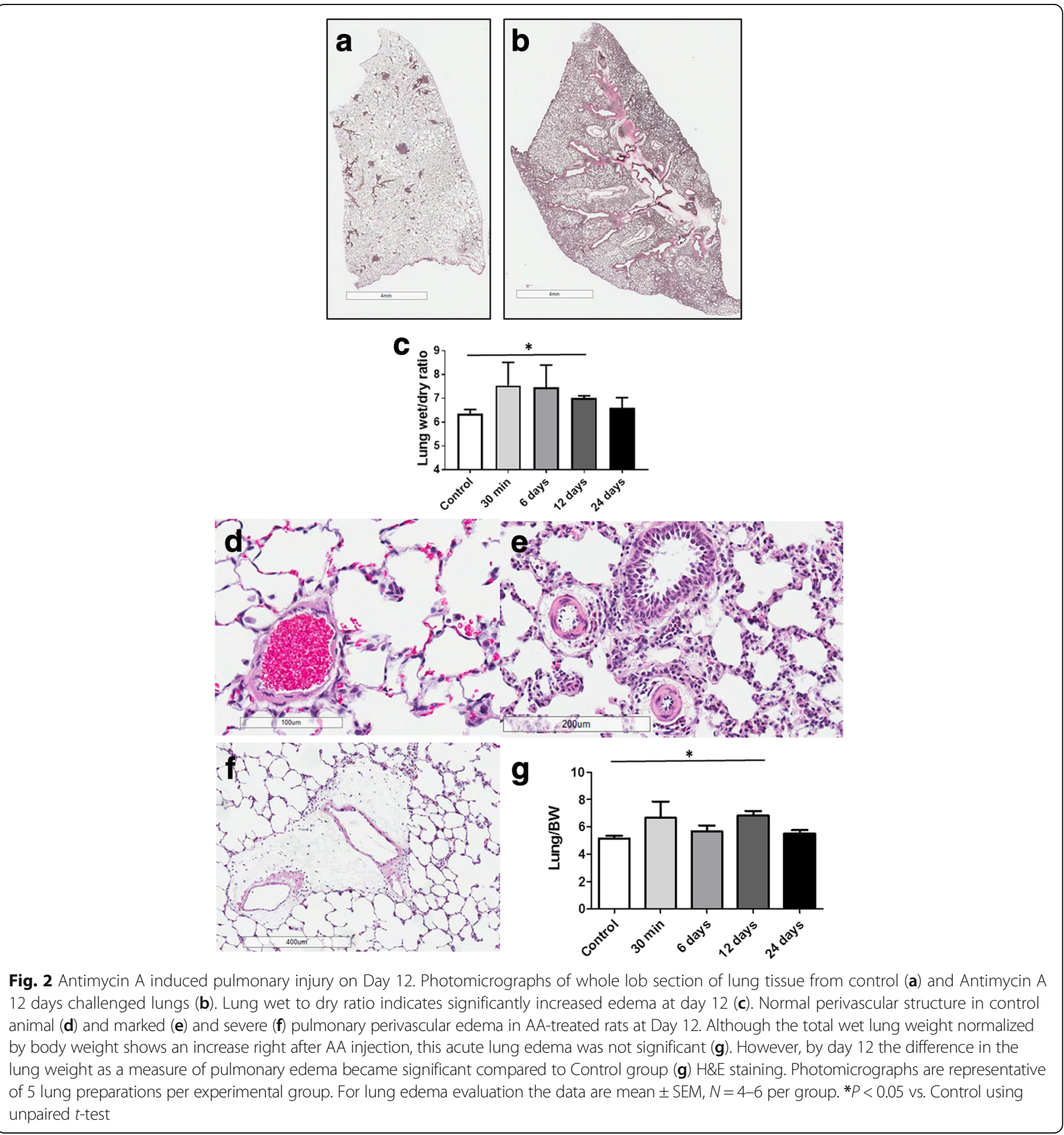




\section{AA treatment produces chronic vasoconstriction and perivascular edema}

The repetitive administration of AA increased the overall size of the lung seen upon examination of the whole lobe section in AA-treated rats compared to Control (Fig. 2a, b), as well as promoted lung vascularization (dark pink staining) and induced severe perivascular edema (light pink areas). Lung wet to dry ratio was increased acutely at $30 \mathrm{~min}$ and chronically at day 6 and 12 , however, significance was reached at day 12 only and was resolved by day 24 (Fig. 2c). Normal perivascular structure in control animal (Fig. 2d) transformed to marked (Fig. 2e) and severe (Fig. 2f) pulmonary perivascular edema in AA-treated rats at day 12. The wet lung weight normalized to the body weight showed an early, but not significant, increase after acute AA injection and the second wave of significant lung weight increase on day 12 (Fig. 2g).

Moreover, the perivascular edema of small pulmonary arteries (negative in control animals Fig. 3a, b) appeared to be a hallmark of the late effect of AA, especially seen on day 12 (Fig. 3c, d, e, f). These results are in great accordance with the changes in the lung weight (Fig. 2f) and lung wet/dry ratio (Fig. 2c).

However, by day 24 perivascular fibrosis, an occasional vascular remodeling and pronounced vasoconstriction continued to progress, as evidenced by Verhoeff-Van Gieson (VVG) staining (Fig. 3g, h). Our quantification of small (Fig. 3i) and large (Fig. 3j) pulmonary arteries perivascular cuffs area at day 12 and 24 exhibited that edema was less evident at day 24 .

The levels of plasma protein nitration correlate with PAH progression in female AA-treated rats and female $\mathrm{PAH}$ patients

The progression of AA-induced pulmonary vasoconstriction was associated with an accumulation of nitrated proteins in the plasma (Fig. 4a). The protein nitration was not evidenced after an acute AA injection and produced only a very mild effect on day 6 , however, at day 12 the increase became significant. Although the statistical significance was not reached in day 24 group due to the high variability among the animals, there was an overall trend of further elevation, suggesting that there may be an association
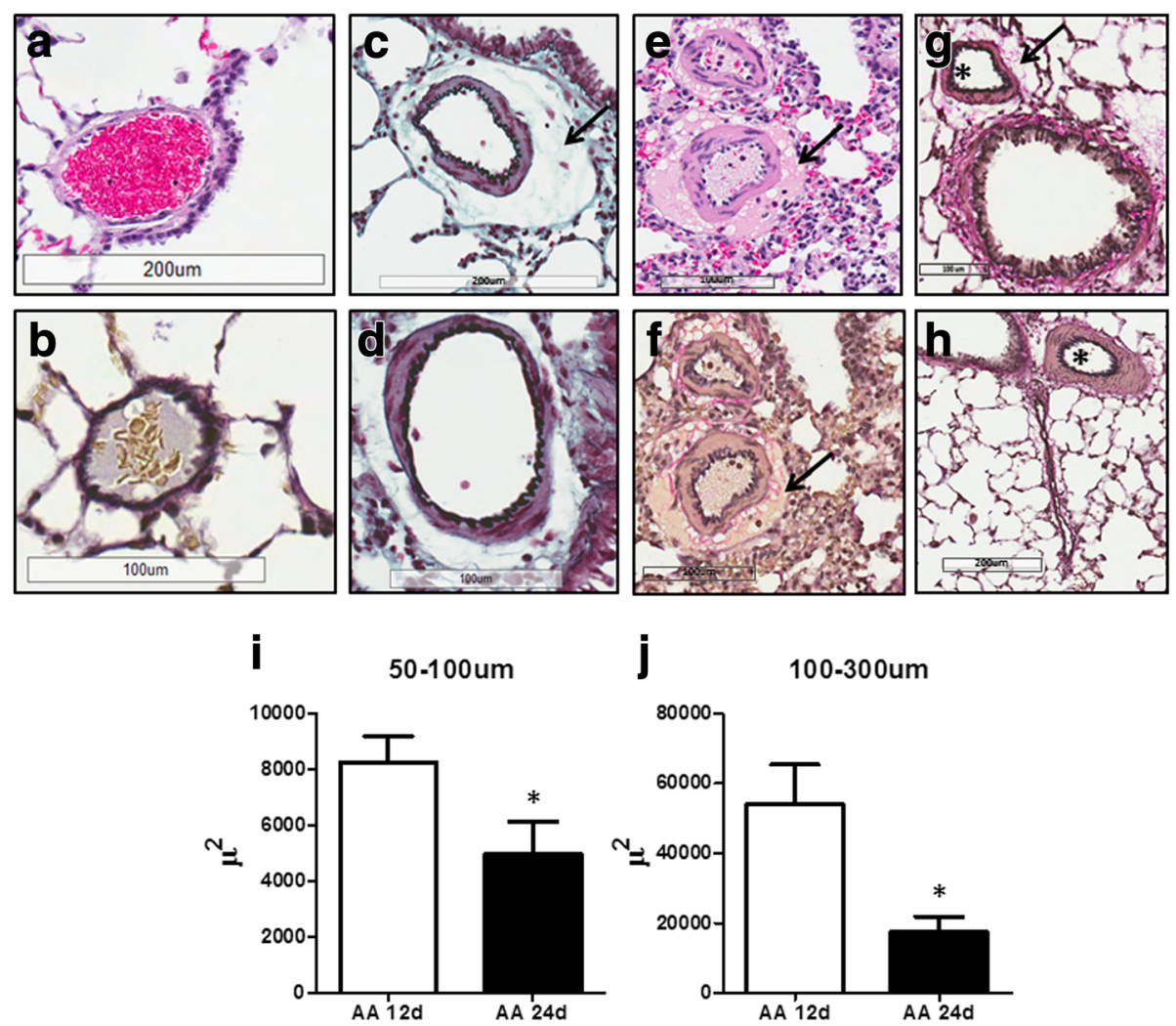

Fig. 3 Small-size pulmonary arteries from Control ( $\mathbf{a}$ and $\mathbf{b}$ ) and Antimycin Day 12 (c and $\mathbf{d}$ ) and Antimycin Day-24 (e, $\mathbf{f}, \mathbf{g}, \mathbf{h})$ rats. Asterisks indicate vasoconstriction and arrows denote the presence of perivascular cuff indicative of endothelial barrier dysfunction. H\&E staining: $\mathbf{a}$ and $\mathbf{e}$; Verhoeff-Van Gieson (WG) staining: b, f-j; Pentachrome staining $\mathbf{c}$ and $\mathbf{d}$. Photomicrographs are representative of 5 lung preparations per experimental group. Scale bar indicate $200 \mu \mathrm{m}$ for $\mathbf{a}, \mathbf{b}$, and $\mathbf{h}$ and $100 \mu \mathrm{m}$ for $\mathbf{c}, \mathbf{d}, \mathbf{e}, \mathbf{f}$, and $\mathbf{g}$. Quantification of perivascular edema area showed a significant decrease in both small (i) and large (j) pulmonary arteries in day 24 group compared with day 12. Data are mean values $\pm S E M, N=9-17$ per group. ${ }^{*} P<0.05$ vs. AA 12 days using unpaired $t$-test 
a
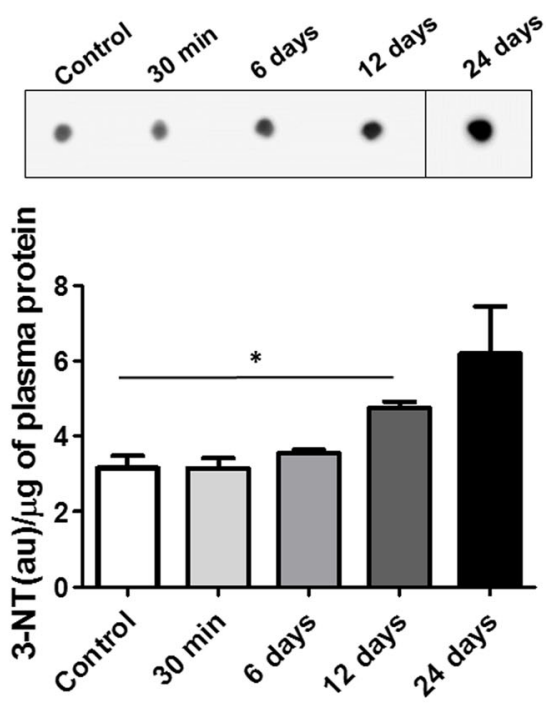

b

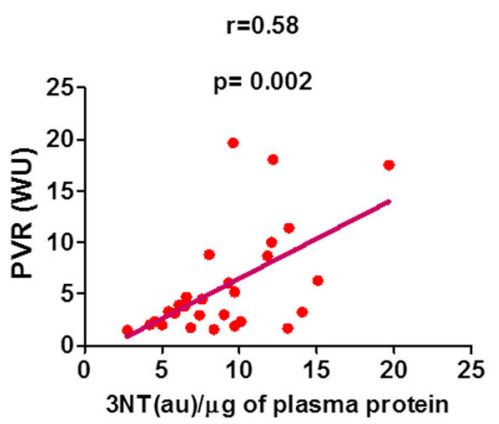

C

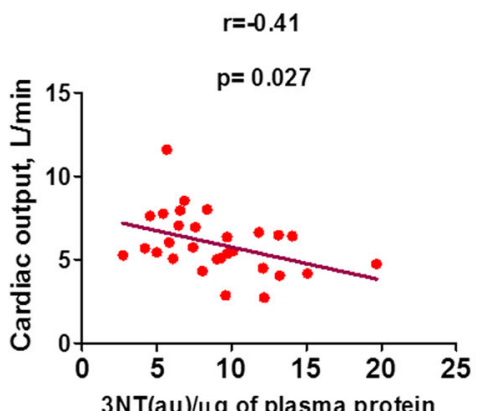

Males

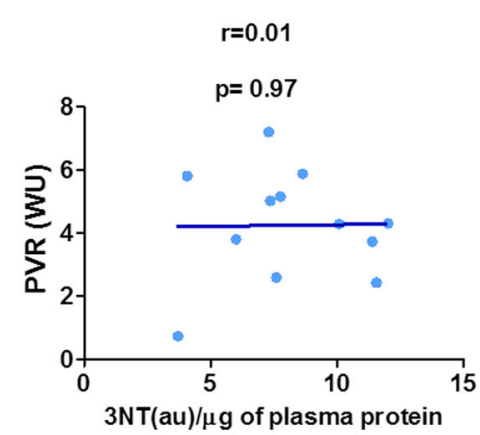

Males

$r=0.1$

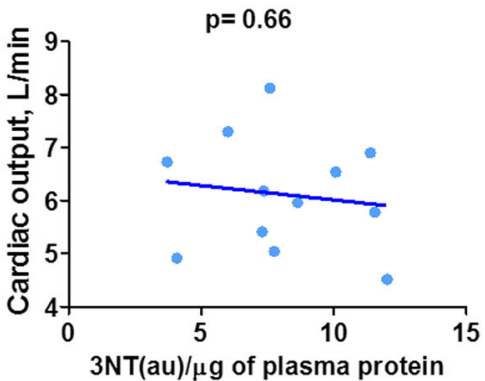

Fig. 4 Total protein nitration in plasma samples of AA-treated rats and from PAH patients. Densitometric analysis indicates that AA injections induced an accumulation of plasma nitrated protein, which become significant by day 12 of study (a). Representative dot for day 24 was taken from different part of the same membrane. The levels of the plasma protein nitration in females but not male patients with PAH positively correlate with pulmonary vascular resistance (PVR, WU, $\mathbf{b}$ and negatively - with cardiac output $(L / \mathrm{min}, \mathbf{c})$, suggesting the nitrosative stress could be involved in $\mathrm{PAH}$ progression in females. $\mathbf{a}$ - data are mean $\pm \mathrm{SEM}, N=3-5$ per group. ${ }^{*} P<0.05$ vs. Control using unpaired $t$-test; $\mathbf{b}, \mathbf{c}-N=12-29$ per group, Person coefficient and $P$-value were calculated by two-tail correlation test

between the levels of protein nitration and PAH progression. Indeed, when the protein nitration was evaluated in PAH patients, it revealed a strong positive correlation with pulmonary vascular resistance (PVR), a key marker of PAH severity, in only female patients (Fig. 4b). There was also a significant negative correlation between nitration of the plasma proteins and a decline in cardiac output $(\mathrm{CO})$ as a measure of $\mathrm{RV}$ 
dysfunction, again, only in female but not male patient groups (Fig. 4c).

\section{Inhibition of mitochondrial respiration produces a severe metabolic shift in lungs}

It has been previously reported that the lungs of both $\mathrm{PAH}$ patients and $\mathrm{PH}$ animals show a metabolic reprogramming and transition to glycolysis [30-33]. To evaluate whether chronic AA injection could mediate the same metabolic shift, we analyzed a few primary metabolites in the lungs of rats on day 6,12 and 24. The analyzed metabolites were specifically chosen to identify changes in the glucose/ribose pathway (Fig. 5) and evaluate the level of glycolytic changes in the lungs after chronic ETC inhibition. We found that all analyzed metabolites possessed a strong increase in the lungs of AA-treated rats. However, the extent and the specific profile of metabolic changes varied at different time points. Thus, there was an increase over 13.9 times in the level of glucose and an increase over 3.4 times in glyceraldehyde 3-phosphate on day 12 compared to control animals (Fig. 6a, b). At the same time the downstream metabolite 3-phosphoglycerate was significantly accumulated only on day 24 (2.2 times increase vs. Controls) and lactate, the final product of glycolysis, was elevated on both 12 and 24 days (2.1 and 2.6 times increase respectively, Fig. 6c, d). Importantly, the pulmonary levels of ribulose-5-phosphate were found to be only decreased

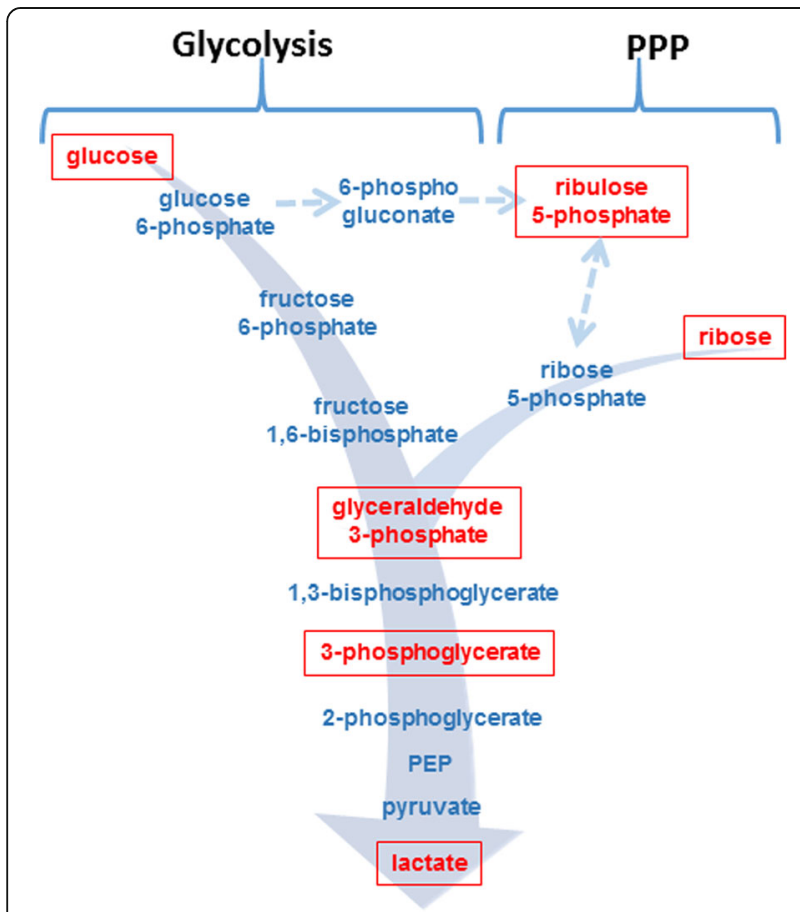

Fig. 5 Analysis of glycolytic switch in Antimycin A-treated rats. To analyze the glycolytic flux in AA-treated rats, we monitored the stable intermediates in the glycolysis and pentose phosphate pathways indicated as red
(Fig. 6e), suggesting that glucose was primarily used in the glycolytic pathway, while the pentose phosphate pathway (PPP) became inhibited (Fig. 5). We found that ribose accumulation (Fig. 6f) may contribute to the glycolysis flux by the shunt into glyceraldehyde 3 -phosphate.

\section{Pulmonary glucose strongly correlates with the level of pulmonary vasoconstriction}

To evaluate whether the discovered strong increase in lung glucose levels was associated with the disease progression, we analyzed the correlation between the lung glucose levels and RVSP in all animal groups together. We found a very robust $(r=0.77)$ and significant $(p<0.0001)$ correlation between these two parameters in our animal model (Fig. 7). These data in a strong accordance with the previously published research proposed that PAH progression is mediated by a shift in the cellular metabolism from glucose oxidation toward glycolysis.

\section{Discussion}

There is a vast body of literature from animal models to patients with $\mathrm{PAH}$ confirming that different types of mitochondrial dysfunction are strongly associated with PAH pathobiology [1, 2, 30, 31]. However, whether these alterations are directly triggered $\mathrm{PAH}$ or subsequent manifestations of the disease and contribute to PAH progression remains unclear. The current study demonstrates, for the first time, that the chronic inhibition of ETC is sufficient to initiate sustained pulmonary vasoconstriction, an increased vascular edema, and a glycolytic metabolic shift in healthy female rats. These observations strongly support the notion that impaired mitochondrial respiration can trigger $\mathrm{PAH}$ development.

Interestingly, when the effect of Crotalaria spectabilis plant seeds given orally was discovered to produce experimental $\mathrm{PH}$ in the 1960 s, the early effects were described as acute pulmonary edema, infiltration of mononuclear cells and eosinophils, and pulmonary arteritis with edema of the pulmonary artery wall $[38,39]$. Pulmonary edema at early stages of PAH (14 days after MCT i.p. injections) was also observed in another recent study, occurring at different anatomic levels (vascular edema, perivascular, bronchial, peribronchial, and alveolar septae) [40]. By day 28 after MCT injection, this edema was found to turn into vascular remodeling, suggesting that MCT induced damage of pulmonary arteries and subsequent edema may contribute to the initiation of uncontrolled vascular repair and remodeling at late stages. In this study, we found that chronic ETC inhibition also induces perivascular edema, which peaks at day 12 and mimics the histological changes seen in MCT and Sugen/hypoxia (our unpublished observation) models. We suggest that 

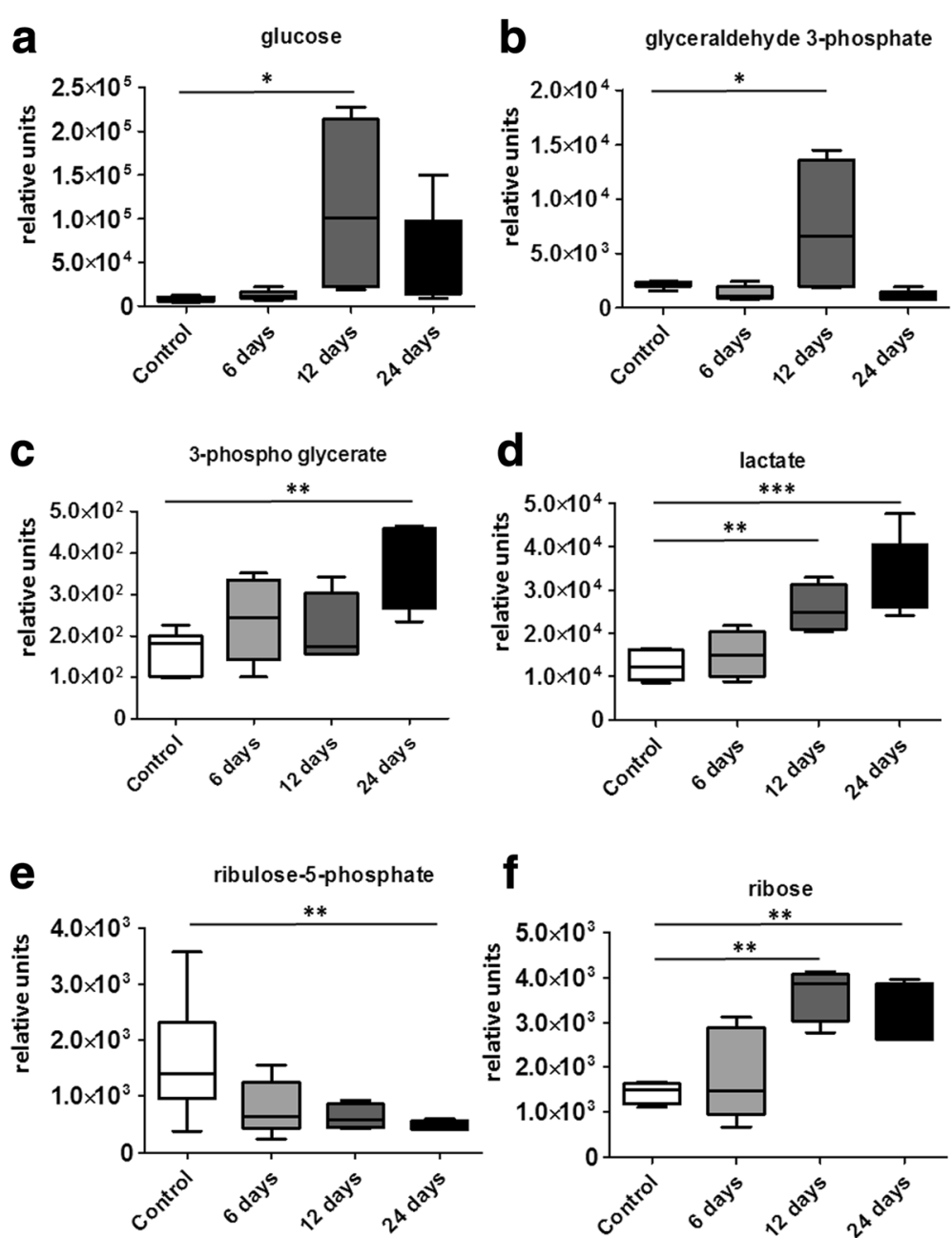

Fig. 6 Chronic electron transport chain inhibition induced a glycolytic shift in lungs. Our data indicate accumulation of glucose (a) and glyceraldehyde 3-phosphate (b) at day 12 of study. In contrast, 3-phosphoglycerate (c) and final product of glycolysis - lactate (d) - possess a progressive increase that becomes significant by day 24 for 3-phosphoglycerate and both day 12 and 24 for lactate. However, an upstream pentose phosphate intermediate - ribulose-5-phosphate (e) has gradually decreased with a disease progression with a significant reduction by day 24. Finally, the content of ribose (f) in the lungs was also strongly elevated at day 12 and 24 , which may represent the ribose to glyceraldehyde-3phosphate shuttle that contributes to upregulation of pulmonary glycolysis. Data are mean \pm SEM, $N=4-7$ per group. ${ }^{*} P<0.05$ vs. Control in 1-way ANOVA

perivascular edema could contribute to vascular stiffness, impair the oxygen transport to the vascular wall and potentiate the activation of inflammatory cells. All these events (vascular stiffness, hypoxia, and inflammatory component) are known to be tightly associated with the increase in the pulmonary arterial pressure and contribute to the pathogenesis of PAH. Besides, this early edema may promote a late onset of vascular proliferation in PAH. Indeed, a subset of pulmonary arteries at days 12 and 24 has displayed the signs of remodeling. These observations support the idea that AA treatment is capable of inducing progressive pulmonary vasoconstriction even 18 days after the last AA application. The development of early lung edema may reflect either a direct deleterious effect of AA on pulmonary endothelial cells or initiated by an enhanced mitochondrial ROS production, which is known to induce p38 MAPK mediated endothelial barrier disruption [41]. Following the histological developments in these rodent models for a longer period of time is likely required to confirm this observation.

In this study, the chronic AA treatment did not result in the development of RV remodeling. While the occasional severe PAH without a detectable RV hypertrophy has been previously described for humans [42] and reported in a mouse model with PAH phenotype [43], the absence of RV hypertrophy did not allow us to consider 


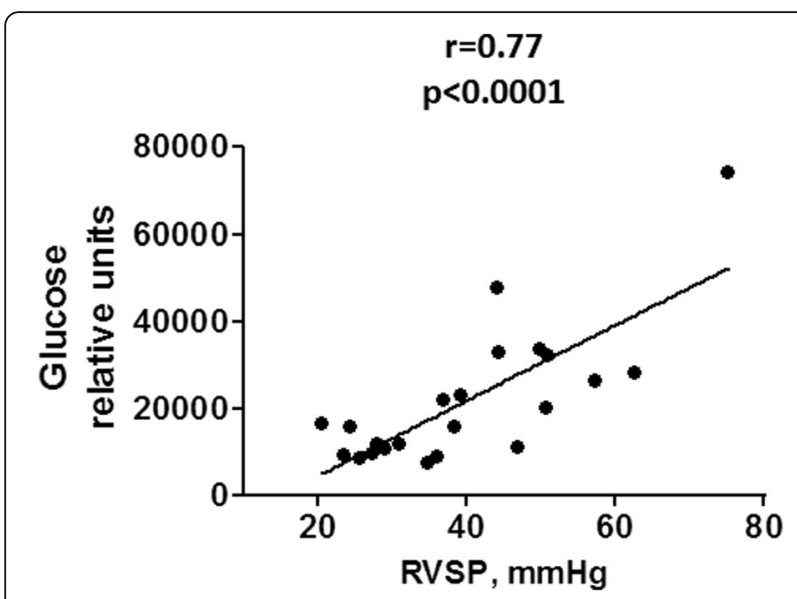

Fig. 7 Correlation between pulmonary glucose levels and right ventricle systolic pressure. The strong correlation between the RVSP and glucose levels in all AA groups analyzed together suggest the contribution of metabolic reprogramming in lungs upon AA treatment in chronic pulmonary vasoconstriction. $N=25$; Person coefficient and $P$-value were calculated by two-tail correlation test

the AA-induced condition as a developed pulmonary hypertension. Instead, we classify the AA-induced changes as an early stage of the disease mainly characterized by chronic pulmonary vasoconstriction. Nevertheless, the absence of RV hypertrophy may be attributed to the inhibitory effect of ETC dysfunction on myocardial contractility. In particular, the reduced levels of ATP production upon ETC inhibition, for example, can diminish RV reserve without an ability to respond to elevated afterload with hypertrophic remodeling. At the same time, the progressively increased RV afterload in combination with reduced RV contractility may be the primary reason for an elevated end-diastolic volume, potentially contributing to an elevated RVDP (Fig. 1c).

The high levels of NO bioavailability in females were expected to induce acute nitrosative stress via an increase in mitochondrial superoxide production induced by ETC inhibition. However, our protein nitration data were not revealing elevations at $30 \mathrm{~min}$ after AA injection. In contrast, there was a progressive accumulation of nitrated plasma proteins that correlated with a gradual disease progression. These data suggest that basal NO production may be insufficient to induce an increase in plasma protein nitration, although it may still produce higher levels of protein nitration in specific cell compartments. However, the progressive chronic vasoconstriction induced by AA treatment may be responsible for the further stimulation of NO production, which, on the background of superoxide leak, could initiate sustained nitrosative stress and may contribute to $\mathrm{PAH}$ progression. This notion was supported by a strong positive correlation between the total protein nitration in the plasma samples of female PAH patients and PVR, and a negative correlation with $\mathrm{CO}$, which both are important markers of PAH severity (Fig. 4). We conclude that the increased level of NO bioavailability associated with female gender promotes accumulation of nitrated proteins specifically in female patients. Nevertheless, whether the observed nitrosative stress contributes to $\mathrm{PAH}$ progression or just could serve as a gender-specific marker of $\mathrm{PAH}$ progression remains to be elucidated.

PAH development and progression are characterized by changes in small circulation hemodynamics, lung and right heart morphology, altered signaling, transcriptomics, proteomics, and metabolism. We have recently reported that before an alteration in macroparameters such as morphology and hemodynamics, we have observed metabolic changes, which predisposed organs to the pathological transition [34]. The glycolytic switch of metabolism in pulmonary vasculature is a well-accepted and characterized phenomenon in $\mathrm{PAH}$. It was shown with a PET scanning of PAH patients that increased glycolysis in lungs and RV, similar to glycolytic changes seen in the tumors of cancer patients, correlates with the presence and severity of PAH $[44,45]$. However, there is still debate whether the glycolytic switch is secondary to electron transport chain dysfunction or is upregulated due to pathologic normoxic HIF1a signaling activation. Our data indicate that ETC inhibition can turn on the glycolytic shift in the lungs. Indeed, AA-induced mitochondria dysfunction led to a robust upregulation of glucose uptake in the lung and resulted in an accumulation of several glycolytic pathway intermediates. Besides, we found that although based on ribulose-5-phosphate data, the PPP pathway was reduced in our animals; the ribose level was upregulated and can be fed into the glycolytic pathway via ribose conversion by ribokinase. It is important to note that glycolysis is elevated 12 days after induction of mitochondrial dysfunction but reduced at the late stage (24 days). Although primary glycolytic pathway is reduced at day 24 , the lactate levels are still elevated. This can be explained by an activation or overexpression of ribokinase that can convert ribose via increased 3PG into production of lactate. Thus, AAinduced mitochondrial dysfunction can lead to the similar metabolic changes observed in other preclinical models and PAH patients.

Aerobic glycolysis is not a very efficient way for ATP generation when compared to the amount produced by mitochondrial respiration. However, the rate of glucose metabolism via glycolysis is higher than the complete oxidation of glucose in the mitochondrial respiratory chain [46]. Moreover, glycolysis induces a carbon flux toward the production of intermediates that are needed for biosynthesis of lipids, amino acids, porphyrins, and 
nucleotides leading to uncontrolled proliferation [47]. An upregulation of glycolysis is known to induce anabolic reprogramming of cells, leading to a higher rate of cell proliferation. Thus, the upregulation of glycolysis in response to mitochondrial dysfunction may become a key factor in vascular remodeling that would maintain the increased pulmonary resistance and pulmonary pressure [48]. It is important to emphasize that glucose uptake by lungs of AA-treated rats was found to strongly correlate with a pulmonary pressure, confirming the importance of glycolysis in PAH progression.

\section{Conclusion}

In conclusion, we believe that this study provides a novel and important insight into the pathogenesis of PAH. The results of our study suggest that the initial mitochondrial dysfunction is involved in the early changes associated with $\mathrm{PAH}$, such as pulmonary vasoconstriction, vascular edema, and metabolic shift. Although these early pathological events have been previously described for other models of PAH, they have never been observed in response to direct mitochondrial inhibition. While further research is required to fully understand the role of mitochondria in the complex mechanisms of PAH initiation and progression, our study provides the novel evidence of the causal role of mitochondrial dysfunction in the initiation of pathological events associated with $\mathrm{PAH}$.

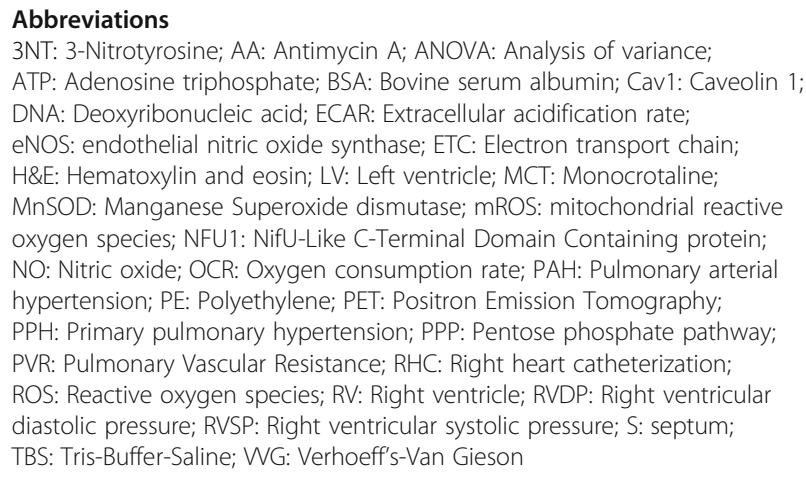

\section{Acknowledgments}

Authors would like to thank Dr. Jason Yuan and Vineet Nair for their contribution in providing the patient's samples to extend available for the clinical study data.

\section{Funding}

This work was supported by NIH grants: 1R01HL133085 (OR), R01HL136603 (AAD) and 1R01HL132918, 14SDG20480354 (RR).

\section{Availability of data and materials}

The datasets used and/or analyzed during the current study are available from the corresponding author on reasonable request.

\section{Authors' contributions}

OR performed experiments, analyzed data and wrote the manuscript, AAD collected and analyzed patient's/clinical data and wrote the manuscript, RR and ST designed the study, analyzed data and wrote manuscript. All authors read and approved the final manuscript.

\section{Ethics approval and consent to participate}

- All subjects provided written consent to participate in this study with the approval of the University of Arizona (UA) institutional human subjects review board. The study cohort consisted of patients with a right heart catheterization (RHC)-confirmed diagnosis of Group I PAH and were prospectively recruited from the UA PAH clinic from 2013 to 2015.

- All experiments were performed in accordance with the University of Pittsburgh Institutional guidelines for animal welfare and the Animal Care and Use Committee approved experimental protocols.

\section{Competing interests}

The authors declare that they have no competing interests.

\section{Publisher's Note}

Springer Nature remains neutral with regard to jurisdictional claims in published maps and institutional affiliations.

\section{Author details}

'Division of Endocrinology, Department of Medicine, The University of Arizona, Tucson, AZ 85724, USA. ${ }^{2}$ Department of Medicine, The University of Arizona, Tucson, AZ, USA. ${ }^{3}$ Vascular Medicine Institute, Division of Pulmonary, Allergy and Critical Care Medicine, Department of Medicine, University of Pittsburgh, Pittsburgh, PA, USA.

Received: 21 December 2017 Accepted: 12 April 2018

Published online: 23 April 2018

\section{References}

1. Freund-Michel V, Khoyrattee N, Savineau JP, Muller B, Guibert C. Mitochondria: roles in pulmonary hypertension. Int J Biochem Cell Biol. 2014;55:93-7.

2. Archer SL, Gomberg-Maitland M, Maitland ML, Rich S, Garcia JG, Weir EK. Mitochondrial metabolism, redox signaling, and fusion: a mitochondria-ROSHIF-1alpha-Kv1.5 O2-sensing pathway at the intersection of pulmonary hypertension and cancer. Am J Physiol Heart Circ Physiol. 2008:294(2):H570-8.

3. Rafikov R, Sun X, Rafikova O, Meadows ML, Desai AA, Khalpey Z, Yuan JX, Fineman JR, Black SM. Complex I dysfunction underlies the glycolytic switch in pulmonary hypertensive smooth muscle cells. Redox Biol. 2015;6:278-86.

4. Saini-Chohan HK, Dakshinamurti S, Taylor WA, Shen GX, Murphy R, Sparagna GC, Hatch GM. Persistent pulmonary hypertension results in reduced tetralinoleoyl-cardiolipin and mitochondrial complex II + III during the development of right ventricular hypertrophy in the neonatal pig heart. Am J Physiol Heart Circ Physiol. 2011;301(4):H1415-24.

5. Xu W, Koeck T, Lara AR, Neumann D, DiFilippo FP, Koo M, Janocha A, Masri FA, Arroliga AC, Jennings $C$, et al. Alterations of cellular bioenergetics in pulmonary artery endothelial cells. Proc Natl Acad Sci U S A. 2007:104(4):1342-7.

6. Afolayan AJ, Eis A, Alexander M, Michalkiewicz T, Teng RJ, Lakshminrusimha S, Konduri GG. Decreased endothelial nitric oxide synthase expression and function contribute to impaired mitochondrial biogenesis and oxidative stress in fetal lambs with persistent pulmonary hypertension. Am J Physiol Lung Cell Mol Physiol. 2016;310(1):L40-9.

7. Pak O, Sommer N, Hoeres T, Bakr A, Waisbrod S, Sydykov A, Haag D, Esfandiary A, Kojonazarov B, Veit $F$, et al. Mitochondrial hyperpolarization in pulmonary vascular remodeling. Mitochondrial uncoupling protein deficiency as disease model. Am J Respir Cell Mol Biol. 2013;49(3):358-67.

8. Adesina SE, Kang BY, Bijli KM, Ma J, Cheng J, Murphy TC, Michael Hart C, Sutliff $\mathrm{RL}$. Targeting mitochondrial reactive oxygen species to modulate hypoxiainduced pulmonary hypertension. Free Radic Biol Med. 2015;87:36-47.

9. Van Houten B. Pulmonary arterial hypertension is associated with oxidative stress-induced genome instability. Am J Respir Crit Care Med. 2015;192(2): 129-30.

10. Diebold I, Hennigs JK, Miyagawa K, Li CG, Nickel NP, Kaschwich M, Cao A, Wang $L$, Reddy S, Chen PI, et al. BMPR2 preserves mitochondrial function and DNA during reoxygenation to promote endothelial cell survival and reverse pulmonary hypertension. Cell Metab. 2015;21(4):596-608.

11. Ryan JJ, Marsboom G, Fang YH, Toth PT, Morrow E, Luo N, Piao L, Hong Z, Ericson K, Zhang HJ, et al. PGC1alpha-mediated mitofusin-2 deficiency in female rats and humans with pulmonary arterial hypertension. Am J Respir Crit Care Med. 2013;187(8):865-78. 
12. Marsboom G, Toth PT, Ryan JJ, Hong Z, Wu X, Fang YH, Thenappan T, Piao L, Zhang HJ, Pogoriler J, et al. Dynamin-related protein 1-mediated mitochondrial mitotic fission permits hyperproliferation of vascular smooth muscle cells and offers a novel therapeutic target in pulmonary hypertension. Circ Res. 2012;110(11):1484-97.

13. Haslip M, Dostanic I, Huang Y, Zhang Y, Russell KS, Jurczak MJ, Mannam P. Giordano F, Erzurum SC, Lee PJ. Endothelial uncoupling protein 2 regulates mitophagy and pulmonary hypertension during intermittent hypoxia. Arterioscler Thromb Vasc Biol. 2015;35(5):1166-78.

14. Olsen LF, Andersen AZ, Lunding A, Brasen JC, Poulsen AK. Regulation of glycolytic oscillations by mitochondrial and plasma membrane H+-ATPases. Biophys J. 2009;96(9):3850-61.

15. Barclay AR, Sholler G, Christodolou J, Shun A, Arbuckle S, Dorney S, Stormon MO. Pulmonary hypertension-a new manifestation of mitochondrial disease. J Inherit Metab Dis. 2005;28(6):1081-9.

16. Xu S, Xu X, Zhang J, Ying K, Shao Y, Zhang R. Pulmonary hypertension as a manifestation of mitochondrial disease: a case report and review of the literature. Medicine (Baltimore). 2017;96(46):e8716.

17. Ahting U, Mayr JA, Vanlander AV, Hardy SA, Santra S, Makowski C, Alston CL, Zimmermann FA, Abela L, Plecko B, et al. Clinical, biochemical, and genetic spectrum of seven patients with NFU1 deficiency. Front Genet. 2015;6:123.

18. Chen Q, Vazquez EJ, Moghaddas S, Hoppel CL, Lesnefsky EJ. Production of reactive oxygen species by mitochondria: central role of complex III. J Biol Chem. 2003;278(38):36027-31.

19. Muller FL, Liu Y, Van Remmen H. Complex III releases superoxide to both sides of the inner mitochondrial membrane. J Biol Chem. 2004;279(47): 49064-73.

20. Slot JW, Geuze HJ, Freeman BA, Crapo JD. Intracellular localization of the copper-zinc and manganese superoxide dismutases in rat liver parenchymal cells. Lab Investig. 1986;55(3):363-71.

21. Rafikova O, Rafikov R, Kumar S, Sharma S, Aggarwal S, Schneider F, Jonigk D, Black SM, Tofovic SP. Bosentan inhibits oxidative and nitrosative stress and rescues occlusive pulmonary hypertension. Free Radic Biol Med. 2013;56:28-43.

22. Tabima DM, Frizzell S, Gladwin MT. Reactive oxygen and nitrogen species in pulmonary hypertension. Free Radic Biol Med. 2012;52(9):1970-86.

23. Fulton DJ, Li X, Bordan Z, Haigh S, Bentley A, Chen F, Barman SA. Reactive oxygen and nitrogen species in the development of pulmonary hypertension. Antioxidants. 2017;6(54). http://www.mdpi.com/2076-3921/6/ $3 / 54$.

24. Bowers R, Cool C, Murphy RC, Tuder RM, Hopken MW, Flores SC, Voelkel NF. Oxidative stress in severe pulmonary hypertension. Am J Respir Crit Care Med. 2004;169(6):764-9.

25. Masri FA, Comhair SA, Dostanic-Larson I, Kaneko FT, Dweik RA, Arroliga AC, Erzurum SC. Deficiency of lung antioxidants in idiopathic pulmonary arterial hypertension. Clin Transl Sci. 2008;1(2):99-106.

26. Zhao YY, Zhao YD, Mirza MK, Huang JH, Potula HH, Vogel SM, Brovkovych V, Yuan JX, Wharton J, Malik AB. Persistent eNOS activation secondary to caveolin-1 deficiency induces pulmonary hypertension in mice and humans through PKG nitration. J Clin Invest. 2009;119(7):2009-18.

27. Rafikov R, Rafikova O, Aggarwal S, Gross C, Sun X, Desai J, Fulton D, Black $\mathrm{SM}$. Asymmetric dimethylarginine induces endothelial nitric-oxide synthase mitochondrial redistribution through the nitration-mediated activation of Akt1. J Biol Chem. 2013;288(9):6212-26.

28. MacRitchie AN, Jun SS, Chen Z, German Z, Yuhanna IS, Sherman TS, Shaul PW. Estrogen upregulates endothelial nitric oxide synthase gene expression in fetal pulmonary artery endothelium. Circ Res. 1997;81(3):355-62.

29. Hisamoto K, Ohmichi M, Kurachi H, Hayakawa J, Kanda Y, Nishio Y, Adachi K, Tasaka K, Miyoshi E, Fujiwara N, et al. Estrogen induces the Akt-dependent activation of endothelial nitric-oxide synthase in vascular endothelial cells. J Biol Chem. 2001;276(5):3459-67.

30. Ryan JJ, Archer SL. Emerging concepts in the molecular basis of pulmonary arterial hypertension: part I: metabolic plasticity and mitochondrial dynamics in the pulmonary circulation and right ventricle in pulmonary arterial hypertension. Circulation. 2015;131(19):1691-702.

31. Paulin R, Michelakis ED. The metabolic theory of pulmonary arterial hypertension. Circ Res. 2014;115(1):148-64.

32. Cottrill KA, Chan SY. Metabolic dysfunction in pulmonary hypertension: the expanding relevance of the Warburg effect. Eur J Clin Investig. 2013;43(8): 855-65
33. Rehman J, Archer SL. A proposed mitochondrial-metabolic mechanism for initiation and maintenance of pulmonary arterial hypertension in fawnhooded rats: the Warburg model of pulmonary arterial hypertension. Adv Exp Med Biol. 2010;661:171-85.

34. Rafikova O, Meadows ML, Kinchen JM, Mohney RP, Maltepe E, Desai AA, Yuan JX, Garcia JG, Fineman JR, Rafikov R, et al. Metabolic changes precede the development of pulmonary hypertension in the Monocrotaline exposed rat lung. PLoS One. 2016;11(3):e0150480.

35. Rafikova O, Rafikov R, Meadows ML, Kangath A, Jonigk D, Black SM. The sexual dimorphism associated with pulmonary hypertension corresponds to a fibrotic phenotype. Pulm Circ. 2015;5(1):184-97.

36. Desai AA, Zhou T, Ahmad H, Zhang W, Mu W, Trevino S, Wade MS, Raghavachari N, Kato GJ, Peters-Lawrence MH, et al. A novel molecular signature for elevated tricuspid regurgitation velocity in sickle cell disease. Am J Respir Crit Care Med. 2012;186(4):359-68.

37. Waypa GB, Marks JD, Guzy RD, Mungai PT, Schriewer JM, Dokic D, Ball MK, Schumacker PT. Superoxide generated at mitochondrial complex III triggers acute responses to hypoxia in the pulmonary circulation. Am J Respir Crit Care Med. 2013;187(4):424-32.

38. Lalich JJ, Ehrhart LA. Monocrotaline-induced pulmonary arteritis in rats. J Atheroscler Res. 1962;2:482-92.

39. Meyrick B, Reid L. Development of pulmonary arterial changes in rats fed Crotalaria spectabilis. Am J Pathol. 1979:94(1):37-50.

40. Bogdan S, Seferian A, Totoescu A, Dumitrache-Rujinski S, Ceausu M, Coman C, Ardelean CM, Dorobantu M, Bogdan M. Sildenafil reduces inflammation and prevents pulmonary arterial remodeling of the Monocrotaline induced disease in the Wistar rats. Maedica (Buchar). 2012;7(2):109-16.

41. Wang T, Chiang ET, Moreno-Vinasco L, Lang GD, Pendyala S, Samet JM, Geyh AS, Breysse PN, Chillrud SN, Natarajan V, et al. Particulate matter disrupts human lung endothelial barrier integrity via ROS- and p38 MAPKdependent pathways. Am J Respir Cell Mol Biol. 2010;42(4):442-9.

42. Suzuki H, HH TT, Sato S. Improvement of pulmonary arterial hypertension following medication and shunt closure in a BMPR2 mutation carrier with atrial septal defect. J Cardiol Cases. 2017;16(1):11-3.

43. Yan L, Chen X, Talati M, Nunley BW, Gladson S, Blackwell T, Cogan J, Austin E, Wheeler F, Loyd J, et al. Bone marrow-derived cells contribute to the pathogenesis of pulmonary arterial hypertension. Am J Respir Crit Care Med. 2016;193(8):898-909.

44. Frille A, Steinhoff KG, Hesse S, Grachtrup S, Wald A, Wirtz H, Sabri O, Seyfarth HJ. Thoracic [18F]fluorodeoxyglucose uptake measured by positron emission tomography/computed tomography in pulmonary hypertension. Medicine (Baltimore). 2016:95(25):e3976.

45. Ahmadi A, Ohira H, Mielniczuk LM. FDG PET imaging for identifying pulmonary hypertension and right heart failure. Curr Cardiol Rep. 2015;17(1):555.

46. Vazquez A, Liu J, Zhou Y, Oltvai ZN. Catabolic efficiency of aerobic glycolysis: the Warburg effect revisited. BMC Syst Biol. 2010;4:58

47. DeBerardinis RJ, Chandel NS. Fundamentals of cancer metabolism. Sci Adv. 2016;2(5):e1600200.

48. Li M, Riddle S, Zhang H, D'Alessandro A, Flockton A, Serkova NJ, Hansen KC, Moldvan R, McKeon BA, Frid M, et al. Metabolic reprogramming regulates the proliferative and inflammatory phenotype of adventitial fibroblasts in pulmonary hypertension through the transcriptional corepressor C-terminal binding Protein-1. Circulation. 2016;134(15):1105-21.

Ready to submit your research? Choose BMC and benefit from:

- fast, convenient online submission

- thorough peer review by experienced researchers in your field

- rapid publication on acceptance

- support for research data, including large and complex data types

- gold Open Access which fosters wider collaboration and increased citations

- maximum visibility for your research: over $100 \mathrm{M}$ website views per year

At BMC, research is always in progress.

Learn more biomedcentral.com/submissions 